University of Nebraska - Lincoln

DigitalCommons@University of Nebraska - Lincoln

Faculty Publications: Materials Research

Science and Engineering Center

Materials Research Science and Engineering

Center

March 2003

\title{
Angular dependence of magnetization reversal process in exchange coupled ferromagnetic/antiferromagnetic bilayers
}

Z.Y. Liu

University of Nebraska - Lincoln

Shireen Adenwalla

University of Nebraska-Lincoln, sadenwalla1@unl.edu

Follow this and additional works at: https://digitalcommons.unl.edu/mrsecfacpubs

Part of the Materials Science and Engineering Commons

Liu, Z.Y. and Adenwalla, Shireen, "Angular dependence of magnetization reversal process in exchange coupled ferromagnetic/antiferromagnetic bilayers" (2003). Faculty Publications: Materials Research Science and Engineering Center. 38.

https://digitalcommons.unl.edu/mrsecfacpubs/38

This Article is brought to you for free and open access by the Materials Research Science and Engineering Center at DigitalCommons@University of Nebraska - Lincoln. It has been accepted for inclusion in Faculty Publications:

Materials Research Science and Engineering Center by an authorized administrator of DigitalCommons@University of Nebraska - Lincoln. 


\title{
Angular dependence of magnetization reversal process in exchange coupled ferromagnetic/antiferromagnetic bilayers
}

\author{
Z. Y. Liu and S. Adenwalla ${ }^{a}$ \\ Department of Physics and Astronomy, University of Nebraska-Lincoln, Lincoln, Nebraska 68588-0111
}

(Received 12 August 2002; accepted 19 December 2002)

\begin{abstract}
The angular dependence of the magnetization reversal process in the exchange biased ferromagnetic/antiferromagnetic bilayers has been investigated carefully using the StonerWohlfarth rotation model. Depending strongly on the orientation of the applied field and the competition between the unidirectional and uniaxial anisotropic energies, the magnetization rotation can occur at either the same side or the different sides of the field direction for the decreasing and increasing field branches of the hysteresis loop. The calculated results and the magnetooptical Kerr effect have been used to understand the magnetization reversal process in the $\mathrm{NiFe} / \mathrm{NiO}$ bilayers, which is caused mainly by the magnetization rotation. (C) 2003 American Institute of Physics. [DOI: $10.1063 / 1.1554760]$
\end{abstract}

\section{INTRODUCTION}

The magnetization reversal process has been one of the interesting issues in magnetic materials. Two basic models, coherent rotation and domain wall motion, are generally used to understand the magnetization reversal. Although the hysteresis loops cannot give direct information about the magnetization reversal, much useful information can still be inferred indirectly. Especially, the magnetization components parallel $\left(M_{\ell}\right)$ and perpendicular $\left(M_{t}\right)$ to the applied field can be determined using proper configurations of the magnetooptical Kerr effect (MOKE) magnetometry ${ }^{1}$ so that a good understanding of the magnetization reversal can be achieved. ${ }^{1,2}$

Recently, a great deal of attention has been given to the magnetization reversal in the exchanged biased ferromagnetic (FM)/antiferromagnetic (AF) systems due to its intriguing phenomena. In the FM/AF systems, the magnetization reversal may drastically change due to the exchange coupling at the interface, which induces many unusual features such as the enhanced coercivity and the loop shift from the origin by an amount known as the exchange field $H_{E} \cdot{ }^{3}$ Recent investigations have shown that, depending on the particular exchange coupled FM/AF system, both the domain wall nucleation and magnetization rotation can be responsible for the magnetization reversal. ${ }^{4-15}$ In this work, the angular dependence of the magnetization reversal process in the FM/AF bilayers has been calculated carefully using the Stoner-Wohlfarth (SW) model, and the calculated results together with the longitudinal and transverse MOKE loops have been used to understand the magnetization reversal in the $\mathrm{NiFe} / \mathrm{NiO}$ bilayers.

\section{EXPERIMENTS}

The sample was prepared in $3 \mathrm{~m}$ Torr Ar pressure using the magnetron sputtering from separate targets. The base

\footnotetext{
a) Author to whom correspondence should be addressed; electronic mail: sadenwal@unlserve.unl.edu
}

pressure was $3 \times 10^{-7}$ Torr. A $45 \mathrm{~nm} \mathrm{NiO}$ layer was first deposited onto the $\mathrm{Si}(100)$ substrate using the rf gun with the deposition rate of $0.034 \mathrm{~nm} / \mathrm{s}$; then a $15 \mathrm{~nm} \mathrm{Ni}_{81} \mathrm{Fe}_{19}$ layer was grown onto the $\mathrm{NiO}$ layer with the deposition rate of $0.024 \mathrm{~nm} / \mathrm{s}$; finally a $10 \mathrm{~nm} \mathrm{Pt}$ layer was coated to prevent oxidation. After the growth, the sample was annealed at a temperature of $252^{\circ} \mathrm{C}$ for $10 \mathrm{~min}$ and then was cooled to room temperature in a magnetic field of $4 \mathrm{kOe}$. The $\mathrm{x}$-ray diffraction shows the polycrystalline texture of the sample.

The magnetization measurements were performed using a MOKE magnetometry. The sample was mounted on a rotatable holder. A $660 \mathrm{~nm}$ laser with $s$ polarization was used as the light source. First, the magnetic field was applied in the plane of the sample and parallel to the incident plane of the light. In this way, the magnetization component $\left(M_{\ell}\right)$ parallel to the field can be determined and the longitudinal MOKE loops of $M_{\ell}-H$ at different angles $\alpha$ between the field and the unidirectional axis were measured. After these measurements, both the sample and the magnet were rotated $90^{\circ}$ with the other parts being fixed. After that, the field was still in the sample plane but perpendicular to the incident plane of the light. By this arrangement, the magnetization component $\left(M_{t}\right)$ perpendicular to the field can be determined and the transverse MOKE loops of $M_{t}-H$ were obtained at different angles $\alpha$. The detailed MOKE arrangement to obtain $M_{\ell}$ and $M_{t}$ has been published elsewhere. ${ }^{1}$

\section{RESULTS AND DISCUSSION}

\section{A. Calculated results}

In the exchange coupled FM/AF bilayers, we assume for simplicity that the unidirectional and uniaxial axes in the FM layer lie along the same direction and the FM layer is in a single domain due to the exchange coupling. Therefore, the magnetization reversal in the FM/AF system may be treated using the Stoner-Wohlfarth (SW) model. ${ }^{16}$ According to the two models proposed by Mauri ${ }^{17}$ and Malozemoff, ${ }^{18}$ domain walls could be formed in the AF layer, which induces the unidirectional interfacial anisotropy and plays the role of de- 


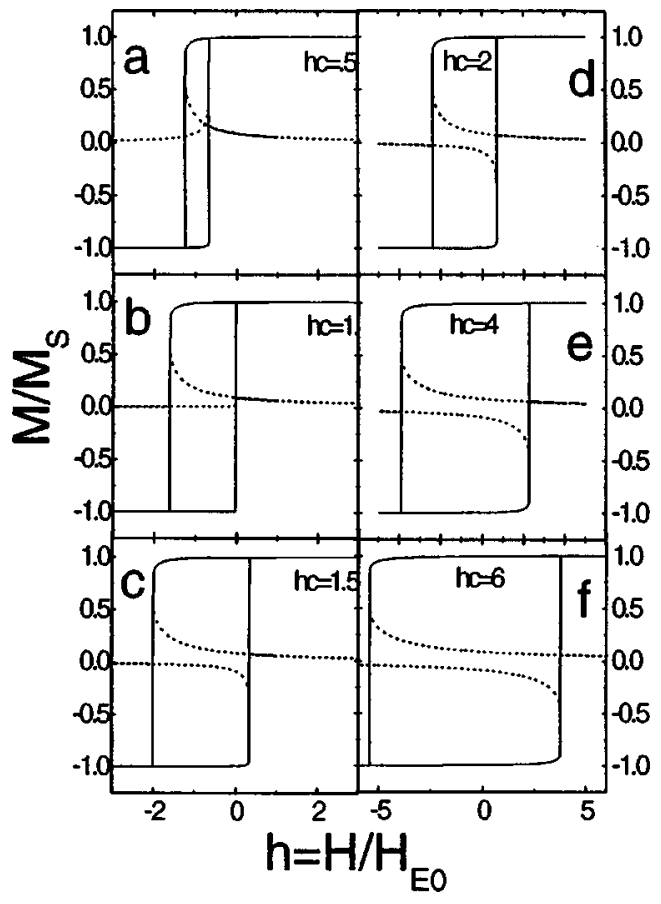

FIG. 1. The calculated $M_{\ell}-H$ and $M_{t}-H$ loops at different values of $h_{c}$ for $\alpha=5^{\circ}$. Solid line: $M_{\ell}-H$ loop; dashed line: $M_{t}-H$ loop.

termining the exchange field of $H_{E} \propto \sqrt{A_{\mathrm{AF}} K_{\mathrm{AF}}}$ with $A_{\mathrm{AF}}$ and $K_{\mathrm{AF}}$ being the exchange constant and the anisotropy of the $\mathrm{AF}$ layer, respectively. The magnetic energy per unit area of the exchange coupled FM/AF bilayers with the thickness of $t_{F}$ and $t_{\mathrm{AF}}$, respectively, can be expressed as ${ }^{10,16}$

$$
\begin{aligned}
E= & K_{F} t_{F} \sin ^{2} \theta_{F}+2 \sqrt{A_{\mathrm{AF}} K_{\mathrm{AF}}}\left(1-\cos \theta_{F}\right) \\
& -H M_{S} t_{F} \cos \left(\alpha-\theta_{F}\right) .
\end{aligned}
$$

The first term is the uniaxial anisotropic energy in the FM layer. The second term is the energy of domain wall formed by rotating interfacial spin of the $\mathrm{AF}$ layer. The last term is the Zeeman energy in the FM layer with a saturation magnetization $M_{S}$. The term $\alpha$ is the angle between the field and the unidirectional axis. $\theta_{F}$ represents the angle of the FM moment with respect to the unidirectional axis. By defining the coercivity $H_{C 0}=2 K_{F} / M_{S}$ and the exchange field $H_{E 0}$ $=2 \sqrt{A_{\mathrm{AF}} K_{\mathrm{AF}}} / M_{S} t_{F}$ along the unidirectional axis and scaling the above energy expression by $H_{E 0}$, Eq. (1) can be written as

$$
\epsilon=h_{c} \sin ^{2} \theta_{F} / 2-\cos \theta_{F}-h \cos \left(\alpha-\theta_{F}\right),
$$

where $h_{c}=H_{C 0} / H_{E 0}$ and $h=H / H_{E 0}$.

The hysteresis loops can be determined by finding out the angle $\theta_{F 0}$ at which the energy is a minimum. Then $M_{S} \cos \left(\alpha-\theta_{F 0}\right)$ and $M_{S} \sin \left(\alpha-\theta_{F 0}\right)$ give the $M_{\ell}-H$ and $M_{t}-H$ loops, respectively.

Figures 1 and 2 give the $M_{\ell}-H$ and $M_{t}-H$ loops at different values of $h_{c}$ for the two field directions of $\alpha=5^{\circ}$ and $70^{\circ}$, respectively. These calculated loops show clearly that the magnetization rotation depends strongly on the values of $h_{c}$ and $\alpha$. At $\alpha=5^{\circ}$ (Fig. 1), the $M_{t}-H$ loops with low values of $h_{c}=0.5,1$ [Fig. 1(a) and 1(b)] reveal that the magnetization rotation occurs at the same side of the field orien-

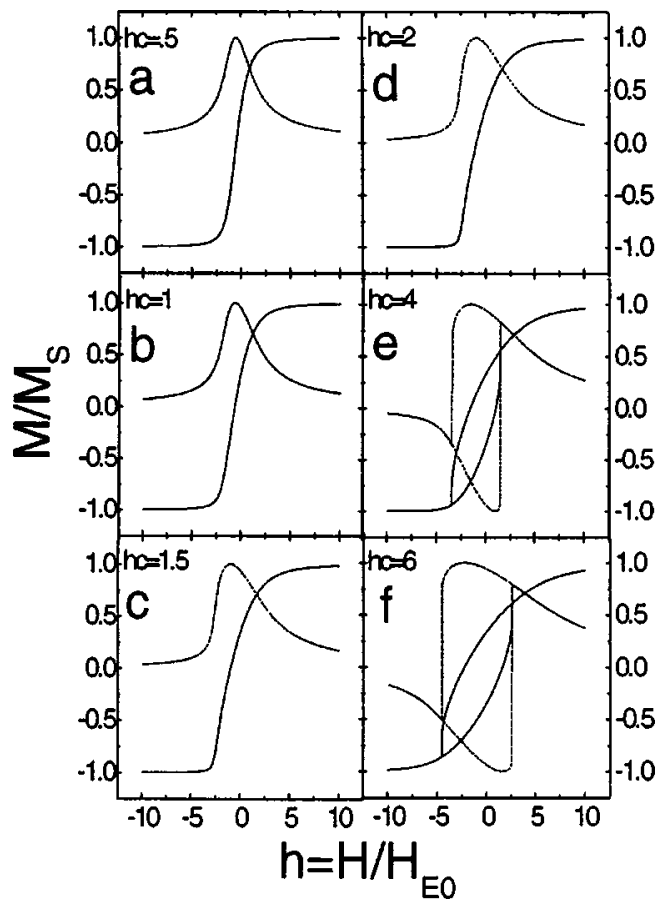

FIG. 2. The calculated $M_{\ell}-H$ and $M_{t}-H$ loops at different values of $h_{c}$ for $\alpha=70^{\circ}$. Solid line: $M_{\ell}-H$ loop; dashed line: $M_{t}-H$ loop.

tation for both the decreasing and increasing field branches of the hysteresis loop, but the $M_{t}-H$ loops with high values of $h_{c}=1.5,2,4,6$ [Figs. 1(c)-1(f)] reveal that the magnetization rotation occurs at the different sides of the field direction for the decreasing and increasing field branches, respectively. This unusual feature of the magnetization reversal cannot be revealed in the $M_{\ell}-H$ loop. Moreover, the shape of the $M_{t}-H$ loop is not symmetric. The lower the value of $h_{c}$, the more obvious the asymmetry of the $M_{t}-H$ loop. With the increasing value of $h_{c}$, the asymmetry of the $M_{t}$ $-H$ loop tends to disappear. As the field is applied close to the hard axis (Fig. 2 at $\alpha=70^{\circ}$ ), only the loops with very high values of $h_{c}=4,6$ [Figs. 2(e) and 2(f)] exhibit the hysteresis. Unlike the $M_{\ell}-H$ loops, the $M_{t}-H$ loops in Figs. 2(e) and 2(f) show the obvious asymmetry of the shape and the magnetization rotation occurring on the different sides of the field orientation. The loops with low values of $h_{c}=0.5,1$, 1.5, and 2 [Figs. 2(a) $-2(\mathrm{~d})$ ] show that the magnetization rotation is reversible and occurs at the same side of the field orientation for both the decreasing and increasing field branches. Unlike the magnetization rotation in the uncoupled FM layer with a uniaxial anisotropy which always occurs at the different sides of the field orientation for the decreasing and increasing branches of the loop, the magnetization reversal in the exchanged coupled FM layer depends strongly on the value of $h_{c}$ and the field orientation $(\alpha)$. The value of $h_{c}$ reflects the relative strength of the uniaxial anisotropy compared to the unidirectional interfacial anisotropy. For a given value of $h_{c}$, there exists a critical angle $\alpha_{0}$, which can be used to determine if the magnetization rotates at the same side or at the different sides of the field orientation for the decreasing and increasing branches. Figure 3(a) gives the value of $\alpha_{0}$ as a function of $h_{c}$. The diagram in Fig. 3(b) 

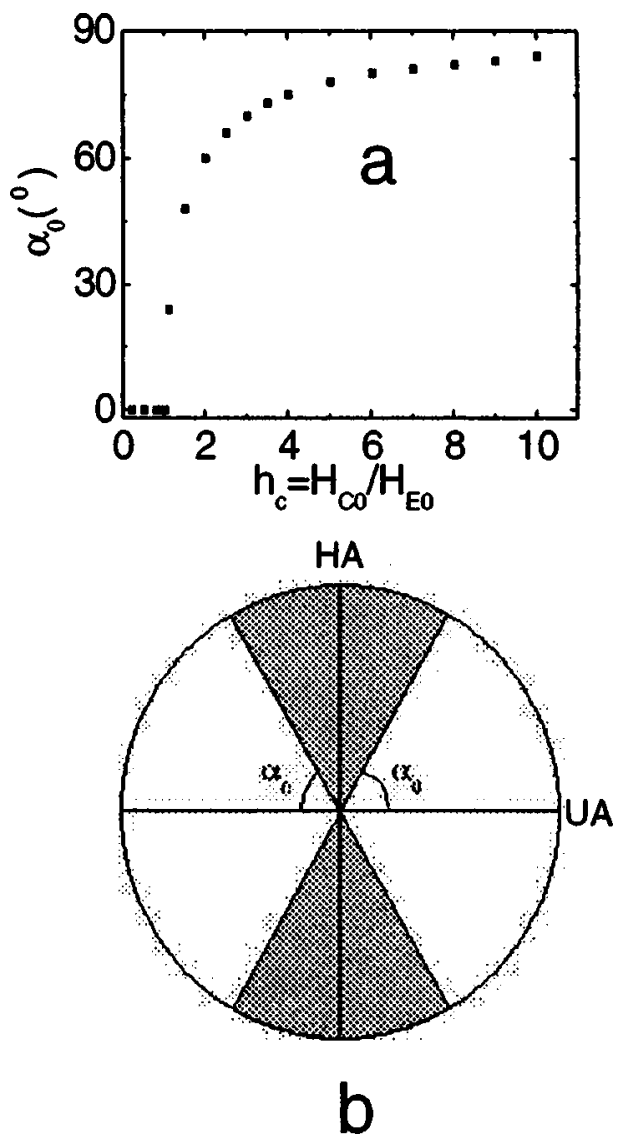

FIG. 3. (a) The critical angle $\alpha_{0}$ as a function of $h_{c}$; (b) the magnetization reversal diagram showing that for a given value of $h_{c}$, the magnetization rotates at the same side of the field direction for the decreasing and increasing field branches of the hysteresis loop when the field is applied in the shadow area, otherwise the magnetization rotation occurs at the different sides of the field direction.

shows that the magnetization rotation occurs at the same side of the field orientation for both the decreasing and increasing field branches when the field is applied in the shadow area; otherwise the magnetization rotation occurs at the different sides of the field orientation for the decreasing and increasing branches. From Fig. 3(a), it can be seen that the critical angle $\alpha_{0}$ is always zero when $h_{c}$ is less than or equal to 1 , implying that the magnetization rotation always occurs at the same side of the field orientation when the unidirectional anisotropy is stronger than or comparable to the uniaxial anisotropy.

\section{B. Experimental results}

Figure 4 gives the experimental and calculated angular dependences of the exchange field $H_{E}$ and coercivity $H_{C}$. The experimental exchange field $H_{E}$ displays the unidirectional symmetry, while the coercivity $H_{C}$ shows the uniaxial symmetry. In the calculation, $h_{c}=0.47$ and $H_{E 0}=45 \mathrm{Oe}$ have been used to get the best simulation to the experimental results. It is shown clearly in Fig. 4 that the calculated angular dependence of $H_{E}$ from the SW model is in good agreement with the experimental result, but the deviation between the experimental and calculated angular dependences of $H_{C}$ is obvious. As the applied field is turned away from the uni-

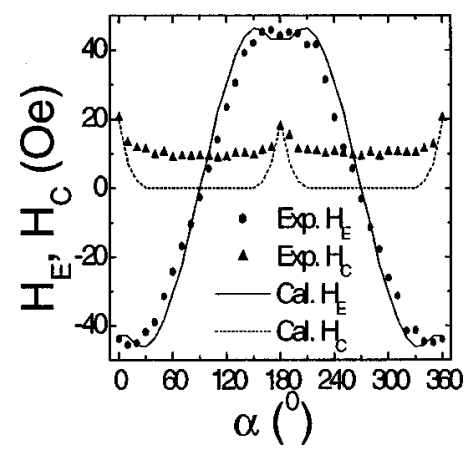

FIG. 4. Both the experimental (exp.) and calculated (cal.) angular dependences of the exchange field $H_{E}$ and coercivity $H_{C}$.

directional axis, the calculated coercivity $H_{C}$ tends to zero swiftly, but the experimental coercivity $H_{C}$ maintains a nonzero value. This feature can be seen clearly in the $M_{\ell}-H$ loops in Fig. 5. With the applied field turning away from the unidirectional axis, the hysteresis of the calculated $M_{\ell}-H$ loop disappears quickly, but the hysteresis of the experimental $M_{\ell}-H$ loop only becomes weaker instead of disappears even when the field is along the hard axis. Even though the SW model cannot account for the angular dependence of the coercivity, it can provide a good simulation to the angular dependence of the exchange field $H_{E}$ and the calculated $M_{\ell}-H$ loops (Fig. 5) still describe the general features of the experimental $M_{\ell}-H$ loops.

According to the calculated results, the low value of $h_{c}$ $=0.47$ suggests that the magnetization always rotates at the same side of the field orientation for both the decreasing and increasing field branch of the hysteresis loops. This feature of the magnetization reversal cannot be revealed by the $M_{\ell}$

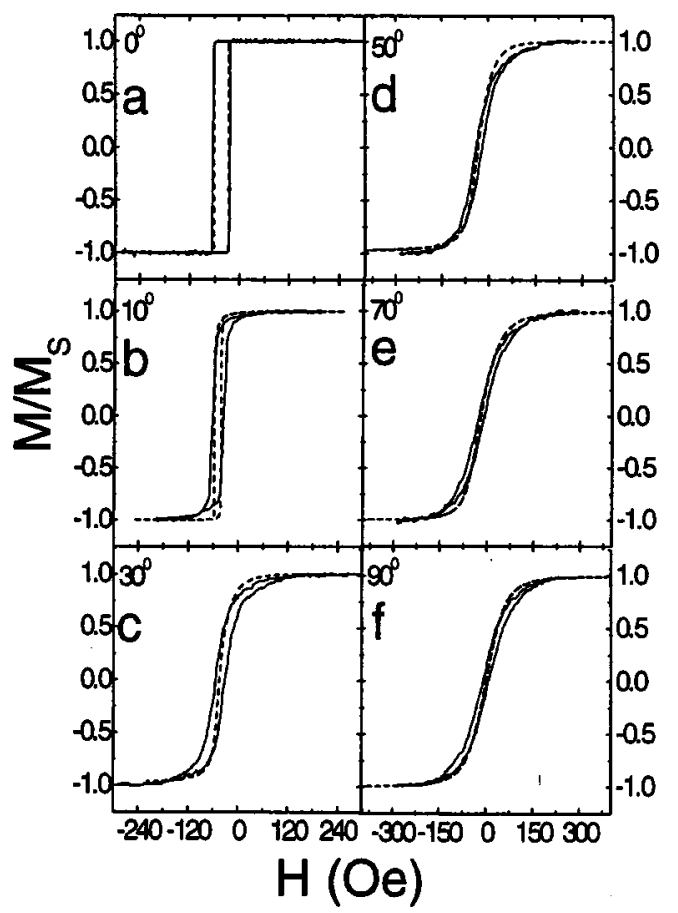

FIG. 5. Both the experimental and calculated $M_{\ell}-H$ loops at different angles of $\alpha$. Solid line: experimental $M_{\ell}-H$ loop; dashed line: calculated $M_{\ell}-H$ loop. In Fig. 5(a), the calculated loop is for $\alpha=2^{\circ}$. 


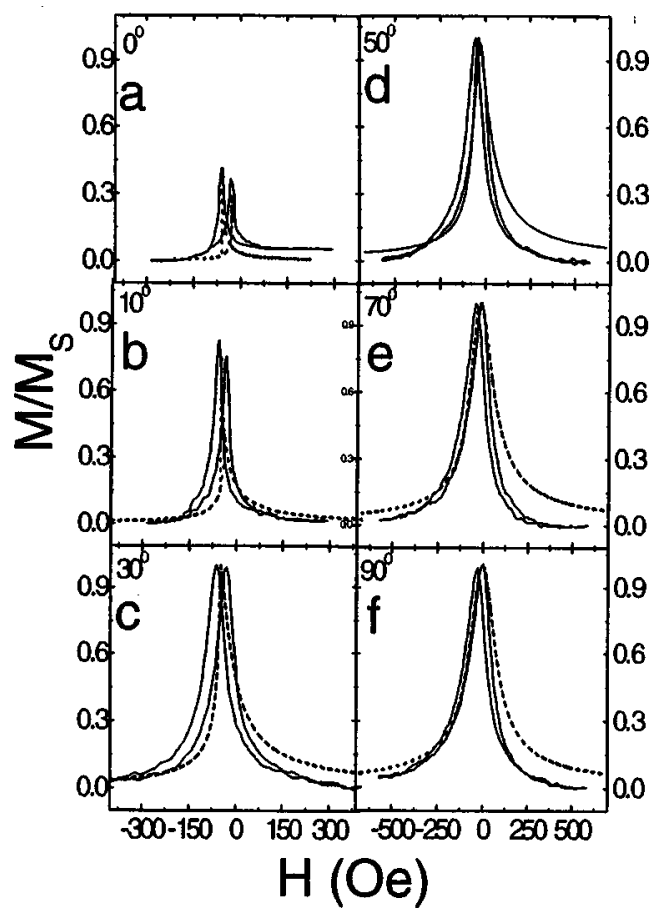

FIG. 6. Both the experimental and calculated $M_{t}-H$ loops at different angles of $\alpha$. Solid line: experimental $M_{t}-H$ loop; dashed line: calculated $M_{t}-H$ loop. In Fig. 6(a), the calculated loops is for $\alpha=2^{\circ}$.

$-H$ loops. Only the $M_{t}-H$ loops can exhibit it clearly. To better understand the magnetization reversal in the $\mathrm{NiFe} / \mathrm{NiO}$ bilayers, the $M_{t}-H$ loops are also measured and shown in Fig. 6 together with the calculated $M_{t}-H$ loops. The calculated $M_{t}-H$ loops cannot describe all the details in the experimental $M_{t}-H$ loops, but they really give the general feature of the experimental $M_{t}-H$ loops: the magnetization reversal in the exchange coupled NiFe layer is mainly through the rotation and the magnetization always rotates at the same side of the field orientation for both the decreasing and increasing branches no matter which direction the field is applied. This feature of the experimental $M_{t}-H$ loops plus the low value of $h_{c}=0.47$ in the calculation imply that the unidirectional interfacial anisotropy is much stronger than the uniaxial anisotropy in the $\mathrm{NiFe} / \mathrm{NiO}$ system. The experimental $M_{t}-H$ loops also display the asymmetry of the shape.

Even though the SW model can describe the main feature of the magnetization reversal in the $\mathrm{NiFe} / \mathrm{NiO}$ bilayers, it cannot explain all features, especially the angular dependence of the coercivity $H_{C}$. In the modified SW model, the domain wall energy in the AF layer is included in the energy expression of Eq. (1), but the domain wall formation and behavior in the FM layer are not considered. In addition to the defects in the FM layer, the domain wall formation in the AF layer may also induce the domain wall formation in the FM layer. ${ }^{12,15}$ In the present $\mathrm{NiFe} / \mathrm{NiO}$ bilayers, the unidirectional anisotropy is much stronger than the uniaxial anisotropy so that the exchange coupling at the interface could pin the domain walls in the FM layer with the magnetization rotation occurring only within domains. However, the presence of domain wall formation can still affect the magneti- zation reversal. Due to the presence of the unidirectional anisotropy, the domain wall formation in the FM layer could be different for the decreasing and increasing field branches of the hysteresis loop. ${ }^{5,11,12,14}$ This difference can cause the asymmetric shape of the hysteresis loop. Therefore, the domain wall formation in both the FM and AF layers may be the main reason for the persistence of the coercivity $H_{C}$ and the asymmetric shape of the $M_{t}-H$ loops in the $\mathrm{NiFe} / \mathrm{NiO}$ system even when the field is applied close to the hard axis.

\section{CONCLUSIONS}

In this article, the angular dependence of the magnetization reversal in the exchange biased FM/AF bilayers has been investigated carefully using the SW model. With the presence of both the unidirectional and uniaxial anisotropies, the magnetization reversal strongly depends on the orientation of the applied field and the competition between the unidirectional and uniaxial anisotropic energies. When the unidirectional anisotropy is stronger than or comparable to the uniaxial anisotropy, the magnetization always rotates at the same side of the field orientation for both the decreasing and increasing field branches of the hysteresis loop. When the uniaxial anisotropy is stronger than the unidirectional anisotropy, a critical field orientation of $\alpha_{0}$, exists. If the field is applied in the ranges of $-\alpha_{0}<\alpha<\alpha_{0}$ and $\pi-\alpha_{0}$ $<\alpha<\pi+\alpha_{0}$, the magnetization rotation occurs at different sides of the field orientation for the decreasing and increasing branches; if the field is applied in the ranges of $\alpha_{0}<\alpha$ $<\pi-\alpha_{0}$ and $\pi+\alpha_{0}<\alpha<2 \pi-\alpha_{0}$, the magnetization rotation occurs at the same side of the field orientation for both the decreasing and increasing branches. This unusual feature of the magnetization reversal can be revealed clearly in the $M_{t}-H$ loop instead of the $M_{\ell}-H$ loop. The calculated results and the experimental $M_{\ell}-H$ and $M_{t}-H$ loops have been used to understand the magnetization reversal in the $\mathrm{NiFe} / \mathrm{NiO}$ bilayers, showing that the SW model can describe the general feature of the magnetization reversal, but cannot explain the angular dependence of the coercivity $H_{C}$, which may be due to the domain wall formation in the exchange coupled NiFe layer.

\section{ACKNOWLEDGMENT}

This work has been supported by NSF9806308.

${ }^{1}$ C. Daboo, J. A. C. Bland, R. J. Hicken, A. J. R. Ives, M. J. Baird, and M. J. Walker, Phys. Rev. B 47, 11852 (1993).

${ }^{2}$ R. P. Cowburn, S. J. Gray, and J. A. C. Bland, Phys. Rev. Lett. 79, 4018 (1997).

${ }^{3}$ J. Nogues and I. K. Schuller, J. Magn. Magn. Mater. 192, 203 (1999).

${ }^{4}$ M. R. Fitzsimmons, P. Yashar, C. Leighton, I. K. Schuller, J. Nogues, C. F. Majkrzak, and J. A. Dura, Phys. Rev. Lett. 84, 3986 (2000).

${ }^{5}$ M. Gierlings, M. J. Pranddini, H. Fritzsche, M. Gruyters, and D. Riegel, Phys. Rev. B 65, 092407 (2002).

${ }^{6}$ T. Gredig, I. N. Krivorotov, and E. D. Dahlberg, J. Appl. Phys. 91, 7760 (2002).

${ }^{7}$ S. Dubourg, J. F. Bobo, B. Warot, E. Snoeck, and J. C. Ousset, Phys. Rev. B 64, 054416 (2001).

${ }^{8}$ T. Mewes, H. Nembach, M. Rickart, S. O. Demokritov, J. Fassbender, and B. Hillebrands, Phys. Rev. B 65, 224423 (2002).

${ }^{9}$ H. Xi, M. H. Kryder, and R. M. White, Appl. Phys. Lett. 74, 2687 (1999).

${ }^{10}$ Z. Qian, J. M. Sivertsen, and J. H. Judy, J. Appl. Phys. 83, 6825 (1998). 
${ }^{11}$ A. Kirilyuk, J. Rasing, H. Jaffres, D. Lacour, and F. Nguyen Van Dau, J. Appl. Phys. 91, 7745 (2002).

${ }^{12}$ V. I. Nikitenko, V. S. Gornakov, A. J. Shapiro, R. D. Shull, K. Liu, S. M. Zhou, and C. L. Chien, Phys. Rev. Lett. 84, 765 (2000).

${ }^{13}$ J. Ding and J. Zhu, J. Appl. Phys. 79, 5892 (1996).

${ }^{14}$ P. Gogol, J. N. Chapman, M. F. Gillies, and F. W. M. Vanhelmont, J. Appl. Phys. 92, 1458 (2002).
${ }^{15}$ P. Steadman, M. Ali, A. J. Hindmarch, C. H. Marrows, B. J. Hickey, S. Langridge, R. M. Dalgliesh, and S. Foster, Phys. Rev. Lett. 89, 077201 (2002).

${ }^{16}$ E. C. Stoner and E. P. Wohlfarth, Trans. R. Soc. London 240, 599 (1948).

${ }^{17}$ D. Mauri, H. C. Siegmann, P. S. Bagus, and E. Kay, J. Appl. Phys. 62, 3047 (1987)

${ }^{18}$ A. P. Malozemoff, Phys. Rev. B 35, 3679 (1987). 